\title{
Ellipsometric study of carbon nitride films deposited by DC-magnetron sputtering
}

\author{
A. Majumdar, ${ }^{1}$ R. Bogdanowicz, ${ }^{1,2}$ and R. Hippler ${ }^{* 1}$ \\ ${ }^{1}$ Institute of Physics, University of Greifswald, Felix-Hausdorff-Str. 6, 17489 Greifswald, Germany \\ ${ }^{2}$ Department of Optoelectronics \& Electronic Systems, Gdansk University of Technology, Narutowicza 11/12, \\ 80-952 Gdansk, Poland
}

Received May 23, 2011; accepted June 17, 2011; published June 30, 2011

\begin{abstract}
We report the optical properties of a carbon nitride $\left(\mathrm{CN}_{\mathrm{x}}\right)$ film as a function of nitrogen concentration $(\mathrm{N} / \mathrm{C})$ of the deposited film. As nitrogen concentration is increased $(\mathrm{N} / \mathrm{C}$ ratio) in a $\mathrm{CN}_{\mathrm{x}}$ film, the refractive index and band gap also increase. The real and imaginary parts, $n$ and $k$ (refractive index and extinction coefficient) of the complex refraction index of carbon nitride films were determined by spectroscopic ellipsometry (SE) in the photon energy range from $1.8 \mathrm{eV}$ up to $4.8 \mathrm{eV}$. Both $n$ and $k$ reveal a significant variation with deposition conditions. A detailed ellipsometric analysis showed that the refractive index varies from 1.8 to 2.05 at different nitrogen concentrations. The optical band gap of the deposited films was situated in the range $1.34 \mathrm{eV}-1.58 \mathrm{eV}$.
\end{abstract}

Carbon nitride films have received considerable attention recently due to their interesting properties (high hardness, high transparency, and chemical inertness) predicted by the early papers of Liu and Cohen [1]. These films have potential applications as hard transparent optical coatings, wear resistant coatings and as a semiconducting material. It has been demonstrated that amorphous semiconducting films can be doped $n$ or $p$ type [2]. The amorphous carbon nitride $\left(\mathrm{a}-\mathrm{CN}_{\mathrm{x}}\right)$ film is expected to be applied widely as a super hard coating material with a low friction coefficient, low band gap protective material on hard disks and read heads, biosensor and anti-biomaterial [37]. In our previous studies we have discussed the influence of nitrogen in bond formation, chemical composition, and surface morphology of $\mathrm{CN}_{\mathrm{x}}$ films [7-9].

In this letter, we report the results of optical measurements on a series of $\mathrm{CN}_{\mathrm{x}}$ samples prepared by magnetron plasma. Optical properties such as refractive index, extinction coefficient and optical band gap have been determined by means of spectroscopic ellipsometry.

An ellipsometric technique is an optical method for thin layer analysis, which is based on polarization state measurement of a light beam reflected form the sample. Such a procedure could be used for thickness monitoring of dielectric and semiconductor layers synthesized during sputtering processes. However, the described procedure has been adapted with respect to that of Tompkins et al.

*E-mail: hippler@physik.uni-greifswald.de
[10], particularly in examining measured data, theoretical modelling, and fitting. Spectroscopic Ellipsometry (SE) data contain quantitative structural information in terms of resonance energies; broadenings of magnitude of absorption bands, especially in the IR and NIR regions (below $8000 \mathrm{~cm}^{-1}$ or $1 \mathrm{eV}$ ); however, SE-data are rarely available in the literature.

Four carbon nitride films were deposited by sputtering a graphite target under different partial pressures (from $3 \mathrm{~Pa}$ to $9 \mathrm{~Pa}$ ) of nitrogen gas in the vacuum chamber. The four different partial pressures are $3 \mathrm{~Pa}, 5 \mathrm{~Pa}, 7 \mathrm{~Pa}$ and $9 \mathrm{~Pa}$. Corresponding to these amounts, $\mathrm{N} / \mathrm{C}$ ratios of the deposited films are $0.11\left(\mathrm{CN}_{3}\right), 0.21\left(\mathrm{CN}_{4}\right), 0.37\left(\mathrm{CN}_{5}\right)$, and $0.55\left(\mathrm{CN}_{6}\right)$, respectively. The $\mathrm{CN}_{\mathrm{x}}$ films were deposited on a p-type $\mathrm{Si}$ (100) substrate which is placed on the ground electrode. The typical film deposition time is 30 minutes for all the deposited $\mathrm{CN}_{\mathrm{x}}$ films. The magnetron discharge power was kept constant for all the deposited samples at 100W.

SE was performed on the samples to study the variation of band-gap energy $E_{g}$ as well as optical properties, including dielectric constants, refractive indices and extinction coefficient of the $\mathrm{CN}_{\mathrm{x}}$ films, under changing process pressure and plasma precursors. Ellipsometric investigations were carried out with a phase modulated ellipsometer Jobin-Yvon UVISEL (HORIBA Jobin-Yvon Inc., Edison, USA) in the photon energy range from $1.8 \mathrm{eV}$ up to $4.8 \mathrm{eV}$ at an angle of $70^{\circ}$, due to Brewster's angle of Si (100) wafer substrate.

A linearly polarized light beam is incident on the sample at $\phi_{0}=70^{\circ}$ angle. For ellipsometric purposes the complex-amplitude parameter $\rho$ is defined as a ratio of total reflection coefficients:

$$
\rho=\frac{r^{p}}{r^{s}}=\tan \Psi e^{j \Delta}
$$

The ellipsometric angles $\Delta$ and $\Psi$ represent the amplitude and the phase ratio difference between complex reflectance in the p-plane and the s-plane of polarization, respectively [10].

The first step of ellipsometric analysis is the assumption of an optical model using a number of layers 
and layer type. Introductory parameterization of optical indices of each layer is necessary to calculate the angles $\Delta$ and $\Psi$ using Eq. (1). The last step of data investigation is finding a model to fit the experimental results. The choice of a suitable optical model is the most critical step. The mean square error (MSE) was utilized as a fitting measure [10], which is given by:

$$
M S E=\frac{1}{2 N-M} \sum_{i=1}^{N}\left[\left(\frac{\Psi_{i}^{\bmod }-\Psi_{i}^{\exp }}{\sigma_{\Psi, i}^{\exp }}\right)^{2}+\left(\frac{\Delta_{i}^{\bmod }-\Delta_{i}^{\exp }}{\sigma_{\Delta, i}^{\exp }}\right)^{2}\right]
$$

In Eq. (2) $\Delta^{\text {exp }}{ }_{i}, \Delta^{\text {mod }}{ }_{i}, \Psi^{\exp }{ }_{i}, \Psi^{\text {mod }}{ }_{i}$ and $\sigma_{i}$ represent the investigated, calculated, and standard deviations at data set $i$, while $N$ is the number of measurement points and $M$ is the number of fitted parameters.

The comparison between measurement results and theoretical model fit, considering the growth of an inhomogeneous layer, allows a quantitative description of the deposited surface. The investigated wavelength region was 400-900nm. Figure 1 displays our results for the ellipsometric angles $\Delta$ and $\Psi$, which are related to the layer thickness $d$ and the optical constants of the film.
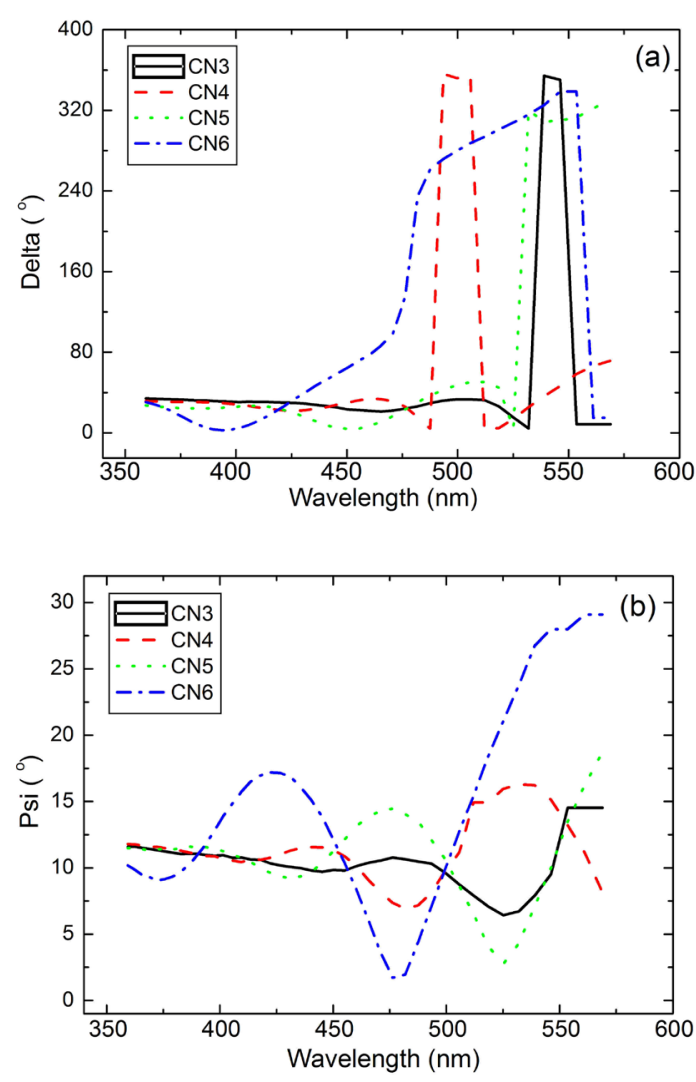

Fig. 1. Ellipsometric angles $\Delta$ (a) and $\Psi$ (b) of $\mathrm{CN}_{\mathrm{x}}$ film deposited on Si substrate versus wavelength.

From Fig. 1, we can see that the phase angle $\Delta$ increases as wavelength increases, indicating that the phase difference or imaginary part of the $\mathrm{s}$ and $\mathrm{p}$ polarizations of light is increasing with a wavelength.

For spectroscopic modelling, it is critical to correctly parameterize the complex dielectric functions of an analysed layer. In this study, a single-layer model based on CN film and substrate is used. The Tauc-Lorentz (TL) dispersion model of dielectric function of a $\mathrm{CN}$ layer was utilized. It was recently proposed for amorphous semiconductors deposited by rf magnetron sputtering. The TL model is a combination of the Tauc joint density of states and the quantum mechanical Lorentz oscillator model [11]. The TL model fits the dielectric functions of amorphous carbide nitride material class, which exhibit a peculiarity due to the presence of two separated contribution of inter-band electronic transition related to $\mathrm{sp}^{2}$ and $\mathrm{sp}^{3}$ bonded carbon [12-13].
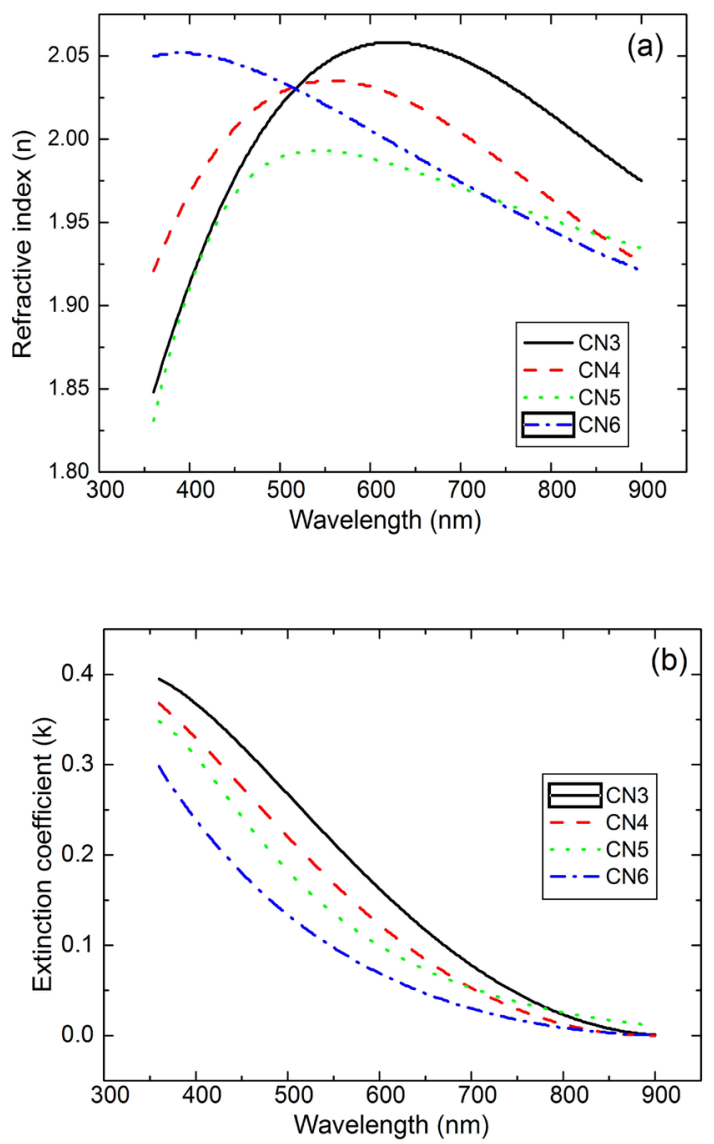

Fig. 2. Optical constants as derived from ellipsometric measurements versus wavelength of the deposited $\mathrm{CN}_{\mathrm{x}}$ films. Real part of the refractive index as derived from a fit based on the TL relation (a) and extinction coefficient as derived from a fit based on the TL relation (b).

In order to extract the optical properties of the deposited films, we assumed that the deposited film 
consists of a single layer of poly-carbonitride on a silicon substrate with a $2.2 \mathrm{~nm}$ thick $\mathrm{SiO}_{2}$ native oxygen layer. The optical indices of Si (100) were obtained from the database [14]. An optical model was fitted to the experimental data, using the Levenberg-Marquardt nonlinear least-squares algorithm. The fitting measure mean square error (MSE) was used to verify the ability of the applied model to fit the experimental data.

Fig. 2 displays the wavelength-dependence of the optical constants $\mathrm{n}$ and $\mathrm{k}$ for the films prepared under different conditions. We note a significant increase in the refractive index with a wavelength up to $610 \mathrm{~nm}$ followed by its gradual decrease above this wavelength. One can also observe the role of nitrogen in the reduction of the refractive index and extinction coefficient. This low value of $\mathrm{n}$ is consistent with the low density of the film. The thickness of the deposited $\mathrm{CN}_{3}, \mathrm{CN}_{4}, \mathrm{CN}_{5}$ and $\mathrm{CN}_{6}$ films is $860 \mathrm{~nm}, 790 \mathrm{~nm}, 720 \mathrm{~nm}$ and $480 \mathrm{~nm}$, respectively.

According to this, $E_{\text {opt }}$ of the films is evaluated from the $\mathrm{x}$-intercept for the linear segment of the plot $(\mathrm{h} v \alpha)^{1 / 2}$ against the photon energy $h v$. Tauc theory shows that $(\mathrm{h} v \alpha)^{1 / 2}$ is proportional to $\mathrm{h} v-E_{\text {opt }}$ for amorphous semiconductors [15]. The electron band gap $E_{\text {opt }}$ was determined by using the Tauc plot.

From Fig. 3, we can see that the optical band gap increases with increasing nitrogen concentration. The role of nitrogen is the key factor in this case. The defect density decreases in the band gap region at a higher nitrogen concentration since the films get less dense. At lower deposition pressure (such as $3 \mathrm{~Pa}$ or $5 \mathrm{~Pa}$ ) the nitrogen concentration is lower and there is a higher probability to form the $\mathrm{sp}^{3}$ bonded carbon materials. At high $\mathrm{sp}^{3}$ fraction films have high internal stress that may create localized states in the band gap and thus contribute to a low optical band gap [16].

$\mathrm{CN}_{\mathrm{x}}$ films with low and/or moderate nitrogen content, i.e., $\mathrm{N} / \mathrm{C}$ ratio $\lesssim 0.35$ display an anomalous dispersion with an increasing index of refraction, and a decreasing absorption coefficient in the range 400-800 $\mathrm{nm}$. By contrast, nitrogen-rich $\mathrm{CN}_{\mathrm{x}}$ films $(\mathrm{N} / \mathrm{C} \geq 0.5)$ display a normal dispersion relation with a falling index of refraction, as well as a falling extinction coefficient.

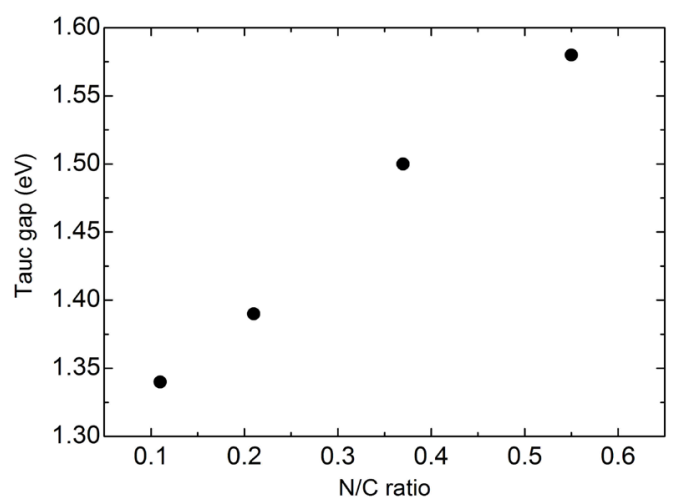

Fig. 3. Optical band gap (Tauc gap) with respect to nitrogen concentration $(\mathrm{N} / \mathrm{C})$ of the deposited $\mathrm{CN}_{\mathrm{x}}$ film.

Part of this work was supported by the Deutsche Forschungsgemeinschaft (DFG) through Sonderforschungsbereich SFB/TR24 "Fundamentals of Complex Plasmas".

\section{References}

[1] A.Y. Liu, M.L. Cohen, Phys. Rev. B 41, 10727 (1990).

[2] F.W. Smith, J. Appl. Phys. 55 (3) 764 (1984)

[3] A. Mansour, D. Ugolini, Phys. Rev. B 47, 10201 (1993).

[4] J. Robertson, Thin Solid Films 383, 81 (2001).

[5] S.P. Lee, Sensors 8, 1508 (2008).

[6] A. Majumdar, K. Schröder, R. Hippler, J. Appl. Phys. 104, 074702 (2008).

[7] A. Majumdar, G. Das, K.R. Basvani, J. Heinicke, R. Hippler, J. Phys. Chem. B 113, 15734 (2009).

[8] A. Majumdar, J. Schäfer, P. Mishra, D. Ghose, J. Meichsner, R. Hippler, Surf. Coat. Technol. 201, 6437 (2007).

[9] A. Majumdar, G. Scholz, R. Hippler, Surf. \& Coat. Technol. 203, 2013 (2009)

[10] H.G. Tomkins, Spectroscopic ellipsometry and reflectometry (Wiley, New York 1999).

[11] G.E. Jellison, Jr, V.I. Merkulov, A.A. Puretzky, D.B. Geohegan, G. Eres, D.H. Lowndes, J.B. Caughman, Thin Solid Films 377-378, 68 (2000).

[12] K.H. Chen, J.-J. Wu, C.Y. Wen, L.C. Chen, C.W. Fan, P.F. Kuo, Y.F. Chen, Y.S. Huang. Thin Solid Films 355-356, 205 (1999).

[13] M. Gioti, S. Logothetidis, Diamond Relat. Mater. 12, 957 (2003).

[14] E.D. Palik ed. Handbook of Optical Constants of Solids (Academic Press, New York 1985).

[15] J. Tauc, R. Grigorovici, A. Vancu, Phys. Status Solidi 15, 627 (1966).

[16] Y. Miyajima, G. Adamopoulos, S.J. Henley, V. Stolojan, Y. Tison, E. Garcia-Caurel, B. Drévillon, J.M. Shannon, S.R.P. Silva, J. Appl. Phys. 104, 063701 (2008). 